Annuaire suisse de politique de développement

$7 \mid 1988$

Annuaire Suisse - Tiers Monde 1987/88

\title{
4. Coopération au développement
}

\section{(2) OpenEdition}

1 Journals

Édition électronique

URL : http://journals.openedition.org/aspd/1339

DOI : 10.4000/aspd.1339

ISSN : 1663-9669

Éditeur

Institut de hautes études internationales et du développement

\section{Édition imprimée}

Date de publication : 1 janvier 1988

Pagination : 115-147

ISSN : 1660-5934

\section{Référence électronique}

«4. Coopération au développement », Annuaire suisse de politique de développement [En ligne], 7| 1988, mis en ligne le 05 avril 2013, consulté le 08 septembre 2020. URL : http://journals.openedition.org/ aspd/1339; DOI : https://doi.org/10.4000/aspd.1339 


\section{Coopération au développement}

Au cours de la période couverte par l'annuaire, le Parlement a examiné trois documents importants de la coopération au développement: le rapport sur la politique de coopération au développement 1976-1985, le message sur le créditcadre de 2'100 millions de francs pour la coopération technique et l'aide financière, ainsique le message sur la participation de la Suisse aux augmentations de capital des banques régionales de développement et sur l'entrée de la Suisse à l'Agence multilatérale de garantie des investissements (AMGI).

Aucune discussion de fond ne s'est engagée au Parlement au sujet du programme de coopération au développement qui a été adopté et confirmé dans sa forme actuelle. Certains avis critiques ont cependant fait valoir qu'il fallait engager les ressources de façon plus efficace et accorder davantage d'attention au secteur privé, ou encore s'orienter beaucoup plus vers les besoins des pays les moins avancés. La politique économique extérieure de la Suisse devrait notamment prendre plus en considération les besoins de la coopération technique.

Les dépenses faites au compte de la coopération au développement rapportent beaucoup à la Suisse: sur chaque franc de la coopération, 94 centimes reviennent en Suisse sous forme de commandes. Cette constatation révèle les vraies motivations de la coopération qui, selon certains intervenants au sein des débats parlementaires, suit ses propres intérêts et s'avère importante pour l'économie suisse d'exportation. L'évolution de la coopération multilatérale est suivie attentivement par certains observateurs qui craignent qu'elle n'empiète sur le terrain de l'aide bilatérale.

\section{Rapport sur la politique de coopération au développement 1976-1985}

La Commission du Conseil national pour les affaires étrangères a présenté un postulat au Conseil fédéral le 9 avril 1985, lui demandant un rapport d'activité sur sa politique de coopération au développement, depuis l'entrée en vigueur de la Loi 
fédérale du 19 mars 1976. Le 2 mars 1987, le rapport a été publié. Il se subdivise en trois parties : problématique du développement, expériences et perspectives des activités de coopération suisse, enjeux et portée de la Loi sur le développement. Le rapport se termine par un aperçu des orientations futures de la politique suisse de développement.

\section{La problématique du développement}

Le domaine d'activité de la coopération technique, tel que la Loi le décrit, consiste à lutter contre la pauvreté et les inégalités dans le monde. Grâce aux apports positifs de la coopération technique internationale, la proportion de pauvres dans la population mondiale a diminué, mais la population du globe croît si rapidement qu'il existe aujourd'hui davantage de pauvres qu'il y a dix ans. La communauté internationale se trouve ainsi face au scandale que représente l'impossibilité de diminuer le nombre absolu de pauvres. Les efforts communs déployés pour les populations défavorisées n'ont pas suffi et devraient être intensifiés, selon le rapport du Conseil fédéral. Par conséquent, l'examen critique du rôle de la coopération technique se justifie pleinement.

Depuis dix ans que la loi est entrée en vigueur, la situation a évolué de façon variable; dans certains pays, la situation s'est améliorée, alors que dans d'autres - elle est pire qu'il y a dix ans. Par exemple, dans les grands pays d'Asie en développement, la production alimentaire a dépassé la croissance démographique, ce qui n'a pas été le cas au Proche-Orient et en Afrique. Les pays en développement sont aussi diversement affectés par la crise de l'endettement.

Parallèlement à la situation des pays en développement, les solutions se sont modifiées au cours des années. La loi suisse a été élaborée au début de 1970,à une époque de confiance dans les solutions économiques globales, de croissance et de division internationale du travail. Dans les années quatre-vingt, cette approche globale a cédé le pas à des tentatives de résoudre les problèmes de chaque pays en développement de façon individuelle et appropriée.

\section{Expériences et perspectives de la coopération technique suisse}

Les projets et programmes de développement de plus d'un million de francs ont été, au cours des dix dernières années, systématiquement examinés par la DDA et l'OFAEE.

L'aide humanitaire et la plupart des crédits mixtes n'ont pas fait l'objet d'un tel examen car les livraisons étaient souvent inférieures à un million de francs.

L'examen s'est fait sous la forme d'un questionnaire pour chaque projet. En tout, 493 projets ont été présentés, $(83 \%$ des projets de la période couverte par le rapport). Le matériel rassemblé a été publié avec le rapport dans un document substantiel. (1) 
L'un des éléments fondamentaux de la coopération technique est la pertinence. Il s'agit là de l'aspect critique que la coopération technique doit englober de façon à apporter une contribution de valeur à un processus de développement durable. La question de la pertinence se rapporte aussi à la répartition des ressources de la coopération technique suisse en fonction de divers critères : répartition géographique et sectorielle, coordination avec les acteurs concernés, intégration dans le processus global.

Les conditions de base de la pertinence de toute activité de développement ont été définies pour la Suisse dans les principes de la coopération au développement. (2)

Au cours de la décennie examinée par le rapport, les dépenses publiques pour le développement ont passé de 281 millions de francs (soit $0,19 \%$ du PNB) à 681 millions de francs $(0,28 \%$ du PNB). Le Conseil fédéral s'adresse des critiques à ce sujet, car ce pourcentage ne reflète ni l'extrême imbrication de l'économie suisse dans le système mondial ni le niveau élevé du produit social brut.

La distribution géographique est fort large puisqu'elle touche 52 des 92 pays en voie de développement. Environ la moitié des projets et $41 \%$ des ressources sont destinés à 19 pays dits prioritaires. Les bureaux de coordination situés dans ces pays doivent faciliter la réalisation des projets.

Les engagements se concentrent sur les secteurs de l'agriculture (27\%), de la formation (16\%) et de la santé (12\%). Au sein de ces secteurs, certains "glissements" se sont produits: la foresterie a gagné en importance, le problème de la sécurité de l'alimentation est étudié et les mesures en matière de santé portent davantage sur les soins de santé primaire que sur les hôpitaux et les cliniques.

Selon les auteurs du rapport, une plus forte concentration des activités suisses de développement aux niveaux géographique et sectoriel serait souhaitable. Depuis quelques années, les programmes de la DDA pour les pays en voie de développement arborent la devise suivante : "Davantage de projets dans moins de secteurs d'activité". Il s'agit d'intensifier les mesures en matière de santé et de population, notamment en zones urbaines (où vit la moitié de la population de certains pays en voie de développement), ainsi que de tenir compte des facteurs écologiques influant sur les projets.

\section{Difficultés des activités liées aux projets}

Les résultats de l'enquête portant sur les difficultés éprouvées dans 364 projets de coopération technique et d'aide financière, ont été les suivants :

4\%: dégâts dus à des catastrophes naturelles,

15\%: problèmes techniques

28\%: obstacles inhérents à la politique sectorielle du pays, voire à la politique économique nationale

37\%: difficultés dans l'environnement socio-économique immédiat du projet

$38 \%$ : problèmes de personnel, difficultés relationnelles entre les collaborateurs 
ou par rapport aux structures administratives

61\%: problèmes de "nature institutionnelle" (par exemple structures juridiques inadéquates, manque de structures organisationnelles bien établies).

Cette énumération des principales difficultés rencontrées illustre l'importance de l'environnement du projet. Afin de mieux intégrer les projets dans le milieu politique et socio-économique, l'expérience montre qu'il faut améliorer le dialogue politique entre le pays en développement et la Suisse, ainsi que la coordination de l'aide entre les donateurs. Cela nécessite de la part des coopérants suisses et des responsables un réel effort de réadaptation aux niveaux organisationnel et personnel.

Les intérêts de la politique de développement doivent occuper une place plus importante dans les relations économiques extérieures.

Afin d'intensifier la pertinence de la coopération suisse, le rapport met l'accent sur l'importance d'intégrer les intérêts de la politique de développement dans l'économie extérieure, plus systématiquement et de façon plus approfondie que jusqu'ici. "La cohérence de cette politique repose essentiellement sur l'intégration des exigences de la politique de développement dans les relations extérieures, notamment économiques; il en va de sa viabilité à long terme. Par ailleurs, la politique de développement doit, en vertu même de son mandat, rechercher des méthodes et des moyens d'intégrer, mieux qu'auparavant, les objectifs de politique extérieure et d'économie extérieure dans son travail." (Rapport, p.62 ").

\section{Répartition des instruments}

Les engagements de la coopération suisse sont en grande partie exempts de conditions de remboursement et de livraison. Les instruments relatifs aux mesures de politique économique et commerciale constituent une exception, de même que l'aide à la balance des paiements et les crédits mixtes. L'aide à la balance des paiements permet de financer, dans le cadre d'un programme de réforme coordonné sur le plan international, des livraisons pour un pays en développement. Jusqu'à présent, environ $80 \%$ de ce type de livraisons provenaient de la Suisse. En ce qui concerne les crédits mixtes, le critère utilisé pour tous les projets suisses consiste à accorder un financement uniquement lorsque les entreprises suisses sont en mesure de présenter une soumission qui soit à un niveau concurrentiel de prix et qualité. (Rapport, p.77 *).

La coopération technique bllatérale est l'instrument prépondérant de la coopération suisse: $71 \%$ des projets et $51 \%$ du volume des activités de développement et coopération de cette dernière décennie se sont inscrits dans ce 
cadre. Des réflexions fondamentales ont été formulées au cours de la période examinée sur la coopération bilatérale. Le premier programme formel de la coopération technique suisse au niveau national a vu le jour en 1973 au Bangladesh, à titre de projet expérimental. Depuis 1985, l'on a élaboré des programmes au niveau des pays pour les pays de la "liste prioritairen.

Quant aux contributions suisses à l'aide multilatérale (1113 millions de francs durant la période du rapport), $40 \%$ ont été destinés à la coopération technique multilatérale et $60 \%$ à des institutions de financement.

Le mot d'ordre de l'aide suisse au développement est "aider autrui à devenir autonome". Cela se traduit par exemple par l'importance donnée aux activités de formation et par la modeste part qu'occupent les activités de production proprement dite; ces dernières ont nettement diminué pendant cette décennie.

Le rapport juge que l'amélioration des conditions de travail des collaborateurs est essentielle pour donner un meilleur rendement aux activités de développement. En effet, la situation de pénurie qui règne au sein du personnel de la DDA et de l'OFAAE a des répercussions sur les méthodes de travail : 17\% des 364 projets de la coopération technique et de l'aide financière, supérieurs à 1 million de francs, connaissent des difficultés en raison du manque de préparation, - un élément qui est demeuré constant pendant ces dix années et n'a pas pu être corrigé.

Le rapport demande également que les offices fédéraux fassent preuve d'une meilleure coordination.

Les principes de la loi sur l'aide au développement demeurent.

En dépit des modifications évidentes de la problématique du développement au cours de la dernière décennie, les principes formulés dans la loi n'ont pas été remis en question, non plus que les méthodes, les objectifs ou le contenu de la coopération technique. La loi s'est révélée comme un cadre bien conçu et efficace pour la coopération; il offre une ouverture suffisante pour les développements ultérieurs.

Les modalités d'exécution de la loi sont stipulées dans l'ordonnance du 12 décembre 1977. Sur la base de cette ordonnance, le Comité interdépartemental pour la coopération internationale et l'aide humanitaire (IKEH) a été créé, mais il n'a guère été utilisé jusqu'à présent. Le Conseil fédéral estime qu'il incombe aux offices chargés des problèmes de politique du développement, d'élaborer au sein de cet organe les aspects de coordination, de conception et autres éléments connexes. II s'agit donc d'activer cet organe; à ce propos, le coordinateur de la Communauté de travail des quatre oeuvres d'entraide privées (3) estime que cette tâche ne pourra être menée à bien que s'il existe une volonté politique de renoncer par exemple dans des cas précis, pour des raisons de politique du développement, à l'octroi de la GRE -dans l'intérêt à long terme de la Suisse; autrement dit, il faudrait sacrifier à court terme les profits de notre économie en vue de construire un monde socialement plus juste. 


\section{Perspectives}

En conclusion, le rapport fait ressortir à nouveau très clairement dans quelle direction la coopération technique suisse devrait être intensifiée:

- "A l'avenir, il s'agit de compléter la promotion en zones rurales par le développement des zones urbaines; la participation de la femme à la coopération technique doit être soutenue, de même que les efforts en matière de politique démographique et la prise en compte des aspects écologiques dans toute activité de développement." (Rapport, p.113).

Le rapport n'analyse pas pourquoi la coopération technique a accordé si peu de place aux femmes jusqu'à présent. Dans les documents annexés au rapport, l'on trouve certaines indications, sous le titre "Aspects de la participation des femmes" : seuls 6 projets de la DDA sur 338 sont réellement des "projets femmes". 23 comportent un volet "femmes". 14 de ces 23 projets ne permettent pas encore de déterminer dans quelle mesure les objectifs relatifs aux femmes ont été atteints.

L'accent continue à être mis principalement sur l'aide bilatérale. D'une manière générale, la politique de développement doit occuper une place plus large dans les relations internationales de la Suisse; cela est considéré comme tout à fait nécessaire.

\section{Débats au Parlement}

Le rapport a été examiné au Parlement pendant la session de juin 1987 (Première chambre, Conseil national) en même temps que le message sur le crédit de programme pour la coopération technique et l'aide financière. La présentation détaillée du rapport et l'autocritique qu'il contient ont été fort appréciées par certains députés. D'autres ont trouvé qu'il n'était pas assez concret et rédigé dans un véritable charabia socio-politique (Eppenberger, PDC); Mme Eppenberger a de plus plaidé en faveur d'une coopération plus étroite avec les organisations privées. M.Renschler (S) a déclaré au nom de son groupe que, compte tenu des objectifs fixés dans la loi, la coopération technique suisse avait pour l'essentiel rempli son mandat. Les points faibles de la coopération technique tiennent en partie à la situation de pénurie de personnel qui limite l'efficacité de l'aide. Il s'agira à l'avenir de mettre d'avantage d'accent sur l'évaluation des projets et programmes.

\section{Remarques:}

1) DDAOFAEE, Documents annexés au Rapport du Conseil fédéral sur la politique de la Coopération technique, 1976 à 1985, Berne, 1987

2) OFAEE, Les principes de la coopération technique suisse, Berne, 4 juillet 1986

3) R.Gerster, Cooperation technique suisse : "Weder Routine noch Kurswechsel", Documentation i3m, juin 1987.

- (version allemande) 


\section{Sources:}

Rapport sur la politique de la coopération au développement, 1976-1985, 2 mars 1987 Documents annexés au rapport du Conseil fédéral sur la politique de coopération au développement, 1976-1985, Berne, 1987

NZZ, 10 et 11 juin 1987

\section{Les dépenses pour l'aide publique au développement en 1986}

Les dépenses au titre de l'aide publique au développement se sont montées en 1986 à 718,3 millions de francs (en 1985 : 681,2). La part de la Confédération représente 706 millions, tandis que les cantons et les communes ont dépensé 12,3

Tableau $n^{\circ} 9$

\section{Aide publique suisse $1985 / 86$ en millions de francs}

1985

1986

Confédération

666,9

706,0

Coopération technique

325,4

325,5

Aide financière

96,3

140.0

Mesures de politique économique et commerciale

73,2

71,8

Aide alimentaire

71,9

53,2

Aide humanitaire

77,5

95,2

Autres contributions'

10,9

10.7

Coúts administratifs

16,1

17,2

Remboursements de préts antérieurs

$-4,4$

$-7,6$

Cantons el communes

14,3

12,3

Coopération technique

8,1

9,9

Aide humanitaire

Total

681,2

718,3

1) Contributions a certaines organisations internationales ne relevant pas du budget de faide suisse, mais pouvant étre incluses dans les montants d'APD selon les directives du $C A D$.

Sources: DDA, Recueil de statistiques, août 1987.

La DDA en 1986. 
millions. Ce poste de dépenses représente $0,28 \%$ du PNB, ce qui est un taux inférieur à la moyenne de $0,36 \%$ des pays membres du CAD. (1)

La plus grande part de l'aide publique au développement est constituée de dons: $95,6 \%$ (en 1985, 95\%); le reste est formé de prêts.

Sur l'ensemble des dépenses de la coopération technique, la DDA et le DFAE ont engagé 610,6 millions de francs, l'OFAEE et le DFEP 71,8 millions, l'Office fédéral des affaires culturelles et le DFI, 3,3 milions. La DDA s'occupe des questions de coopération technique, aide financière et aide humanitaire. Quant à I'OFAEE, il traite principalement des mesures de politique économique et commerciale. Le DFI se charge avant tout des bourses. Les frais administratifs se sont montés à 17,2 millions de francs.

Les différentes opérations de coopération technique menées par la Confédération sont financées par des crédits-cadre. (L'énumération des crédits-cadre en vigueur pendant l'année du rapport se trouve dans l'Annuaire 1986, p.104). La répartition des mesures d'aide au développement prises par la Confédération, les cantons et les communes, figure sur le tableau $\mathrm{N}^{\circ} 9$.

La répartition de la coopération au développement en aide bilatérale et aide multilatérale correspondait pendant l'année du rapport pratiquement à celle des années précédentes : 178,1 millions de francs, ou 24,8\% (en 1985: 21,8\%) pour les mesures multilatérales et 540,2 millions, ou $75,2 \%$ (en $1985: 78,2 \%$ ) pour l'aide bilatérale.

Le tableau $N^{\circ} 10$ ci-après présente les dépenses d'aide multilatérale et bilatérale:

Tableau $n^{\circ} 10$

Répartition de la coopération au développement de la DDA en alde bilatérale et aide multilatérale en1986 en millions de francs

\begin{tabular}{|c|c|c|c|c|}
\hline & bilatérale & multilatérale & total & $\%$ \\
\hline Coopération technique & 250,5 & 71,7 & 322,2 & \\
\hline Aide financière & 105.1 & 34,9 & 140,0 & \\
\hline total & 355,6 & 106,6 & 472,2 & 75,7 \\
\hline Aide alimentaire & 43,3 & 9,9 & 53,2 & \\
\hline Aide humanitaire & 44,3 & 50,9 & 95,2 & \\
\hline total 1. & 87,6 & 60,8 & 148,4 & 24,3 \\
\hline Total & 443,2 & 167,4 & 610,6 & 100,0 \\
\hline
\end{tabular}

Source: DDA, Recueil de statistiques 1986. 
La part de l'aide multilatérale, et particulièrement les cofinancements avec l'AID, a connu une augmentation régulière au cours de ces dernières années. Certains acteurs de la coopération au développement observent cette tendance avec un sentiment de malaise car ils estiment que la Suisse n'a pas élaboré jusqu'à présent un véritable concept de l'aide multilatérale.

\section{Coopération technique et aide financière - nouveau message}

\section{2'100 millions de francs pour les trois prochaines années}

La coopération technique et l'aide financière sont du point de vue quantitatif les deux volets essentiels de l'aide publique au développement de la Suisse. Ces ressources servent à financer en premier lieu des projets et des programmes en faveur des pays en développement les plus pauvres et des secteurs de la population les moins favorisés.

Le Conseil fédéral propose dans un message au Parlement un nouveau crédit de programme de 2' 100 millions de francs qui remplacera probablement dès le 31 octobre 1987 le crédit de programme en cours de 1'800 millions de francs, engagé à partir du 1 er novembre 1984.

Le message a été présenté en même temps que le rapport sur la politique en matière de coopération au développement de 1976 à 1985. II met par conséquent l'accent sur la description du programme qui doit être réalisé à travers le nouveau crédit de programme; en ce qui concerne l'exposé des expériences antérieures, il renvoie au rapport proprement dit.

Le Conseil fédéral constate que, en principe, la coopération au développement occupe une place importante dans la politique extérieure suisse. II rappelle les lignes directrices de la politique suisse : neutralité, disponibilité, solidarité et universalité. Hormis l'amélioration de la situation des pays en voie de développement, la coopération au développement favorise également les intérêts économiques suisses. L'aide au développement stimule l'économie suisse de manière directe: en 1985, l'on a estimé à 629,3 millions de francs les achats de biens et services suisses financés par des fonds d'aide au développement, alors que l'aide publique au développement atteignait 666,9 millions de francs. Si l'on ajoute à cela les achats effectués en Suisse par des pays en développement grâce à des crédits de la Banque mondiale, on obtient pour 1985 un montant total de 1'312,7 millions de francs. (Message, p.6).

L'expansion de la coopération au développement se justifie, selon le Conseil fédéral, par la détérioration de la situation de nombreux pays en développement en raison de la crise économique et de la chute des prix des matières premières. La Suisse tient à soutenir les efforts des pays en voie de développement, à les aider à compter sur leurs propres forces. Le Conseil fédéral constate que les conditions d'octroi de fonds pour le développement se sont durcies. Les mesures 
d'ajustement structurel sont par exemple devenues souvent une condition préalable à l'attribution de l'aide, ce qui a entrainé parfois des coûts sociaux élevés. La part des fonds versés sous forme de dons n'augmente guère; l'aide destinée aux pays les moins avancés a même diminué au cours des dernières années.

Le Conseil fédéral évoque aussi le rôle des pays industrialisés qui devraient opérer les restructurations nécessaires au sein de leur propre économie afin d'accéder à une politique économique qui favorise une croissance plus équilibrée de l'économie mondiale.

\section{L'aide bilatérale: élément principal}

Le nouveau crédit de programme porte sur la période 1987 à 1989. Sur les ressources prévues, $71 \%$ doivent être destinés aux pays les moins avancés; le taux correspondant dans le dernier crédit était de $67 \%$. La part qui est dévolue aux pays à revenu intermédiaire est de $9 \%$, alors qu'elle était de $10 \%$ dans la période précédente.

La coopération suisse au développement continue à reposer principalement sur la coopération technique et l'aide financière bilatérales. Environ $70 \%$ de l'ensemble des ressources disponibles sont utilisées pour la coopération bilatérale, alors que $30 \%$ sont consacrés à l'aide multilatérale.

Cette répartition des fonds a passé pendant longtemps pour une ligne de conduite inamovible. Toutefois, les statistiques sur lesquelles cette ligne s'appuie ont donné lieu à des critiques de la part des oeuvres d'entraide:"*

II semble que la Confédération cofinance de plus en plus des projets et programmes spécifiques d'institutions qui figurent dans les statistiques sous la rubrique de l'aide bilatérale. La plupart de ces accords de cofinancement sont conclus avec l'AID, filiale de la Banque mondiale; en 1985, les montants versés à l'AID ont représenté 26,1 millions de francs, sur les 90,9 millions attribués aux organisations internationales (cf. Tableau 3.3. p. 345 dans l'Annuaire 1986). M.Gerster a évalué à $47 \%$ en moyenne le montant destiné à la coopération multilatérale au cours de la période 1981 à 1985 , et non pas $28 \%$ seulement, comme le Conseil fédéral l'avait affirmé en 1986 dans sa réponse à l'interpellation de M.Belser, conseiller aux Etats. Selon l'avis de M.Gerster, la tendance à l'accroissement de l'aide multilatérale persiste, ce qui est problématique, car cette forme d'aide n'est que faiblement implantée parmi la population, alors que l'aide

- La classification des groupes de pays utilisée ici correspond à celle de l'OCDE:

- 36 PMA (pays les moins avancés)

- autres pays à bas revenu (PNB par habitant inférieur à 700 \$)

- pays à revenu moyen (PNB par habitant supérieur à 700 \$).

** R. Gerster, coordinateur de la Communauté de travail Swissaid, Action de Carême, Pain pour le prochain, Helvetas, Coopération suisse pour le développement : "Weder Routine noch Kurswechsel", Documentation i3M, Juin 1987. 
bilatérale directe inspire davantage confiance. En outre, l'orientation de l'aide suisse envers les pays les moins avancés plaide en faveur de l'augmentation de l'aide bilatérale.

Les pays occidentaux industrialisés consacrent en moyenne $72 \%$ des fonds de coopération à l'aide bilatérale (1985), ce qui est inférieur à la coopération bilatérale suisse, dans le cadre de laquelle la part destinée aux pays à bas revenu atteint $88 \%$. Selon M.Gerster, il manque une véritable conception de l'aide multilatérale. II dénonce le fait que, au cours des dernières années, le Conseil fédéral n'a pas exposé clairement dans ses nombreux messages et rapports, les priorités en matière de coopération multilatérale. De temps à autre, l'aide multilatérale est justifiée en bloc, comme c'est le cas dans le message dont il est question ici. Il manque notamment une comparaison détaillée des institutions, par exemple au sujet de la participation de la Suisse aux processus de décision. Les oeuvres d'entraide font valoir les raisons qui plaident en faveur de l'aide multilatérale: il est impensable de négliger la coopération multilatérale pour résoudre certains problèmes.

\section{Distribution des ressources}

Sur les ressources engagées bilatéralement, $46 \%$ sont réservés à l'Afrique, $38 \%$ à l'Asie et $16 \%$ à l'Amérique latine. Néanmoins, c'est l'Afrique au sud du Sahara qui demeure le principal bénéficiaire. $69 \%$ de l'aide est destinée à 18 pays en tête de liste des bénéficiaires, soit 10 en Afrique, 5 en Asie et 3 en Amérique latine.

Cette répartition géographique va rester sans doute semblable à l'avenir; la coopération va continuer à se concentrer sur un nombre de domaines relativement peu élevé. En première place figure l'agriculture dont le rôle va être intensifié. D'une manière générale, il faut accorder plus d'attention à la contribution de la femme et à son rôle dans le processus de développement, notamment dans les projets portant sur le développement social.

II s'agit de promouvoir la coopération avec les organisations non gouvernementales qui sont en général proches de la population avec laquelle elles collaborent étroitement en vue d'améliorer leur situation socio-économique. Toutefois, les partenaires directs les plus importants demeureront, même à l'avenir, les services gouvernementaux du pays bénéficiaire concerné.

Le message évoque aussi la nécessité d'augmenter le personnel de la DDA. De 1976 à 1985, le volume moyen des projets gérés par chaque collaborateur a passé de 1 à 3,2 millions de francs. Il faudrait également intensifier la coordination entre les divers offices fédéraux chargés de la coopération au développement, particulièrement entre la DDA et l'OFAEE.

De plus, l'évaluation doit être améliorée, car elle représente un instrument indispensable pour affiner les activités de développement. Un accent tout particulier devrait être mis sur les projets de cofinancement avec l'AID et d'autres institutions régionales d'aide financière, de même que sur les différentes formes de coopération avec des institutions des Nations Unies. 


\section{La continuité}

Dans l'ensemble, le message est fort détaillé, particulièrement en ce qui concerne l'orientation à long terme et la continuité de la coopération suisse au développement qui n'ont jamais été si clairement exposées. La plus grande partie des ressources est utilisée pour des projets et programmes déjà entamés. Pour la première fois, le message du Conseil fédéral contient des indications sur la part du budget consacrée aux actions courantes et celle consacrée aux nouvelles actions. Dans un seul pays par exemple, le Tchad, qui est un des pays prioritaires au Sahel, la moitié environ des 3,1 millions de francs annuels est destinée aux nouveaux projets. Dans les 17 autres pays de la liste prioritaire, cette proportion est au maximum de $25 \%$, et en général de $10 \%$ ou moins.

\section{Recherche en matière de développement}

La recherche scientifique dans le domaine de la coopération au développement doit être stimulée par des fonds tirés du crédit de programme. "Il s'agit d'élaborer des concepts et des stratégies de coopération pour les années à venir, voire jusqu'en l'an 2000." (Message, p. 60 de la version allemande). Il est prévu de consacrer environ 3,5 millions de francs par an au financement de cette recherche.

\section{Information de l'opinion publique}

Malgré ses 25 ans d'existence, la coopération au développement en tant qu'institution permanente de la politique suisse n'est pas encore entrée dans les moeurs d'une grande part de l'opinion publique. Une information bien diffusée devrait sensibiliser la population à la problématique du développement. Les dépenses prévues à cet effet sont d'environ 1,5 million de francs par année.

\section{Le Parlement adopte le crédit}

Le nouveau crédit de programme a été adopté sans opposition par le Conseil national et le Conseil des Etats qui ont examiné le dossier au cours de leurs sessions respectives de juin et septembre 1987. Le soutien apporté aux efforts des pays en développement a été considéré comme un élément fondamental d'une politique étrangère solidaire. M.Renschler (S) a exhorté le Parlement à évaluer de façon réaliste ce crédit de 2,1 milliards, car ce montant qui apparaît certes élevé, pourrait faire croire à des largesses. En réalité cependant, la Suisse fait de bonnes affaires avec l'aide au développement, puisque des sommes considérables sont ensuite dépensées pour l'achat de biens et services en Suisse. 


\section{Sources}

Messsage concernant la continuation de la coopération technique et de l'aide financière en faveur des pays en développement, 2 mars 1987

R. Gerster, Coopération suisse au développement: "Weder Routine noch Kurswechsel", i3m, Juin 1987.

$\mathrm{NZZ}, 10$ et 11.6 .1987

\section{Aide bilatérale}

La part la plus importante de l'aide publique au développement est composée de mesures bilatérales. L'aide publique bilatérale va du gouvernement du pays donateur au gouvernement du pays bénéficiaire. Cette forme d'aide est bien implantée en Suisse et on la préfère à l'aide multilatérale. Cette attitude s'est traduite dans de nombreuses votations populaires. Le refus du peuple suisse à propos de l'adhésion à l'ONU peut aussi être interprété comme une volonté de privilégier le contact direct avec le pays partenaire.

L'aide bilatérale de la Suisse (y compris l'aide associée) a représenté en 1986 540,2 milions de francs, soit $75,2 \%$ de l'ensemble de l'APD. Ce chiffre englobe les dépenses bilatérales de la coopération technique, l'aide financière, les mesures de politique économique et commerciale ainsi que l'aide humanitaire, comprenant l'aide alimentaire. Les projets de cofinancement avec l'AID pour un montant de 60 millions de francs constituent une part importante de ce total.

Les dépenses par régions figurent au tableau $\mathrm{N}^{\circ} 11$ :

Tableau $n^{\circ} 11$

\section{Alde bllatérale de la Confédération en 1985/86; répartitlon par réglons}

1985

1986

Régions

millions de frs.

$\%$

millions de frs.

$\%$

Afrique

Amérique latine

269,9

50,7

289,5

76,2

53,6

Asie/Ocóanie

82,0

15,4

112,3

14,1

Europo

121,7

22,9

20,8

Non spécifié

0,3

$-0,5$

62.7

$-0,1$

11,0

11,6

Total

532,5

100,0

540,2

100,0

Source: DDA, Recueil des statistiques 1986. 
Pour l'aide bilatérale, la région de concentration est l'Afrique; avec $53,6 \%$ de l'aide totale, ce continent a reçu davantage que lors des années précédentes. Les contributions au titre de l'aide humanitaire (aide alimentaire comprise) constituent une part non négligeable (44,2 millions de francs). Les projets régionaux en Afrique ont bénéficié du plus fort soutien : 36 millions de francs, dont environ 3 millions pour les pays du Sahel. L'aide distribuée se présente de la manière suivante : Tanzanie (34,6 millions), Madagascar (21,2), Sénégal $(20,6)$, Burundi (19,3), Rwanda (18,5), Bénin $(11,2)$ et d'autres pays. L'Afrique a toujours été un des plus importants bénéficiaires de l'aide suisse au développement.

Selon la classification par groupes de pays, les pays les moins avancés (36 pays en voie de développement d'après l'OCDE) ont reçu en $198637 \%$ de l'aide bilatérale, les autres pays en développement à bas revenu (jusqu'à 700 dollars de PNB par habitant) $28 \%$, et les pays en développement à revenu moyen, $22 \%$ de l'aide. Le tableau ci-après donne un aperçu de cette répartition:

Tableau $n^{\circ} 12$

Classement des pays bénéficlaires de l'alde bilatérale selon le revenu

\begin{tabular}{|c|c|c|c|c|c|c|}
\hline & \multicolumn{2}{|c|}{1984} & \multicolumn{2}{|c|}{1985} & \multicolumn{2}{|c|}{1986} \\
\hline & Mio Fr. & $\%$ & Mio Fr. & $\%$ & Mio Fr. & $\%$ \\
\hline MA & 166,0 & 34 & 172,2 & 33 & 198,6 & 37 \\
\hline utres PVD a bas revenu & 172,2 & 36 & 171.4 & 32 & 153,7 & 28 \\
\hline PVD a revenu moyen & 60,3 & 12 & 80,6 & 15 & 70.0 & 13 \\
\hline (y compris frais administ & 86,5 & 18 & 108,3 & 20 & 177.9 & 22 \\
\hline tol & 485,0 & 100 & 532,5 & 100 & 540,2 & 100 \\
\hline
\end{tabular}

Souce: DDA, Recueil de statistiques 1986, aout 1987.

La DDA a consacré environ $58 \%$ des dépenses bilatérales, soit 355,6 millions de francs, à la coopération technique et à l'aide financière. Ces dépenses se sont réparties pendant les deux dernières années dans les secteurs économiques énumérés ci-dessous : 
Tableau $n^{\circ} 13$

Secteurs économiques

1985

1986

\begin{tabular}{|c|c|c|c|c|}
\hline & Mio Fr. & $\%$ & Mio Fr. & $\%$ \\
\hline $\begin{array}{l}\text { Agrlculture } \\
\text { Production agricole } \\
\text { Sylviculture, arboriculture } \\
\text { Irrigation, services agricoles } \\
\text { Elevage } \\
\text { Autres }\end{array}$ & $\begin{array}{r}109,4 \\
44,0 \\
24,3 \\
16,4 \\
7,5 \\
17,2\end{array}$ & 33,4 & $\begin{array}{l}89,9 \\
32,8 \\
17,1 \\
17,3 \\
7,8 \\
14,9\end{array}$ & 25,3 \\
\hline $\begin{array}{l}\text { Industrio } \\
\text { Industries lieses a lagriculture } \\
\text { Autres industries }\end{array}$ & $\begin{array}{r}28,6 \\
9,1 \\
19,5\end{array}$ & 8,7 & $\begin{array}{r}34,9 \\
8,4 \\
26,5\end{array}$ & 9,8 \\
\hline Communications, transports, & 35,0 & 10,7 & 38,9 & 10,9 \\
\hline Commerce, banques, & 29,7 & 9,1 & 23.4 & 6.6 \\
\hline Administration publique & 0,5 & 0,1 & 20,7 & 5,8 \\
\hline $\begin{array}{l}\text { Educatlon } \\
\text { Enseignement universitairo } \\
\text { Enseignement technique su } \\
\text { perieur } \\
\text { Alphabetisation et autres } \\
\text { formations }\end{array}$ & $\begin{array}{l}37,6 \\
12,7 \\
8,1 \\
16,8\end{array}$ & 11,5 & $\begin{array}{l}34,8 \\
11.5 \\
7.6 \\
15.7\end{array}$ & 9,8 \\
\hline Santo, hyglene, services & 30,1 & 9,2 & 270 & 7,6 \\
\hline Wultisocteurs't & $\begin{array}{l}31,8 \\
16,0\end{array}$ & 9,7 & 66,5 & $187 \%$ \\
\hline Non clases 6 & 25,0 & 76 & 19,5 & 5.5 \\
\hline Total & 327,7 & 100,0 & 355,6 & 100,0 \\
\hline
\end{tabular}

1) Projets régionaux do développement rural, ressources naturelles et humaines, développement communautaire, planification.

Source DDA informations communiqués en aout 1987 . 
La répartition géographique de la coopération technique et de l'adie financière de la DDA montre que l'accent est de nouveau mis en 1986 sur l'Afrique : 197,8 millions de francs ou $55,7 \%$. L'Amérique latine représente une part de $15 \%(53,5$ millions de francs) et l'Asie, de 21,1\% (75,2 millions de francs).

La coopération technique est en général financée par des dons; elle a pour but d'accroître les connaissances et capacités des pays en développement dans le domaine technique. II s'agit par exemple d'améliorer les capacités des pays en développement à utiliser les facteurs de production de façon plus rationnelle. L'aide est dispensée sous forme de projets et de programmes dans le domaine technique.

L'aide financière est également constituée de dons, mais elle comprend parfois aussi des prêts et accompagne la coopération technique. L'aide financière est accordée dans divers secteurs à des fins d'investissement et est souvent destinée à des programmes sectoriels ou d'ajustement structurel.

Les dix-sept premiers pays destinataires de l'aide publique bilatérale au développement (1986)

Tanzanie

Madagascar

Burundi

Sénégal

Bolivie

Rwanda

Inde

Indonésie

Népal

Cameroun

Mali

Honduras

Bangladesh

Bénin

Guinée-Bissau

Kenya

Niger

Projets régionaux en Afrique

Projets régionaux en Asio

Projets régionaux en Amérique centrale et du sud

Autres pays 164,9

Non spécifié (1)

TOTAL
34,6 millions de francs

21,2

19,3

20,6

18,9

18,5

18,3

17,5

16,4

14,8

14,8

11,8

11,2

10,6

10,6

10,5

9,7

16,0

7,9

12,2

50,5

540,2 millions de francs

1) Versements concernant plusieurs pays

Source: La DDA en 1986, Annexe 
Cette liste est sujette à d'importantes modifications chaque année car l'attribution de crédits pour la balance des paiements ou de crédits mixtes échoit à des moments différents, ce qui influence fortement les statistiques.

\section{Alde multilatérale}

L'aide multilatérale est accordée aux pays en voie de développement par le biais d'une organisation internationale qui s'occupe de développement. En 1986, l'APD multilatérale de la Suisse s'est montée à 178,1 millions de francs, soit $24,8 \%$ de l'APD totale.

En ce qui concerne la Suisse, les principaux partenaires de l'APD multilatérale sont le Programme de développement des Nations Unies (PNUD), l'Oeuvre d'entraide pour l'enfance (UNICEF), les Banques de développement africaine, asiatique et interamériciane, la filiale de la Banque mondiale (AID), le Programme alimentaire mondial (PAM), le Haut-Commissariat des Nations Unies pour les réfugiés (HCR) et le Comité international de la Croix-Rouge (CICR). Le tableau $N^{\circ}$ 14 donne un aperçu des contributions fédérales aux organisations internationales.

En matière de choix des institutions, les critères doivent être conformes aux "principes de l'aide suisse au développement" du 4 juillet 1986, c'est-à-dire une politique de développement accordant une grande importance à l'amélioration des conditions de vie des secteurs de la population défavorisés et à la diminution de la dépendance des pays en voie de développement par rapport à l'étranger, ainsi que l'efficacité de l'institution et la possibiité pour la Suisse de participer pleinement aux processus de décision. Toutefois, l'analyse comparative et l'évaluation des institutions cofinancées par la Suisse, sur la base des critères susmentionnés, n'existe pas encore.

Au cours de la période examinée, les débats relatifs à l'aide multilatérale ont surtout porté sur les documents des banques régionales de développement et l'adhésion de la Suisse à l'AMGI. (Ce dernier point est traité au Chapitre 7.5. du présent Annuaire).

\subsection{Banques régionales de développement}

Dans un message au Parlement, le Conseil fédéral a proposé en novembre 1986 que la Suisse participe à l'augmentation de capital de la Banque interaméricaine du développement (BID), de la Banque asiatique du développement (ADB) et de la Banque africaine du développement (BAD). Dans le même message, le Conseil fédéral traite de l'adhésion de la Suisse à l'Agence multilatérale de garantie des investissements (MIGA). Ce sujet est traité au chapitre des institutions financières internationales. Le crédit de programme proposé s'élève à 680 millions de francs, 
Tableau $n^{\circ} 14$

Contributions fédérales aux organisations internationales (en millions de francs)

Organisations

1986

1. Organisations des Nations Unies (N.U)

110,5

Programme des N.U. pour le développement (PNUD)

58,6

Programme alimentaire mondial (PAM)

4,4

Office des N.U. pour les réfugiés de Palestine (UNRWA)

8,4

Fonds des N.U. pour l'enfance (UNICEF)

13,0

Haut Commissariat des N.U. pour les réfugiés (HCR)

5,0

Fonds des N.U. pour les activités en matière de

population (UNFPA)

5,5

Organisation mondiale de la santé (OMS)

3,5

Autres organisations

12,1

2. Banques réglonales de développement et leurs fonds speciaux

Banque africaine de développement (BAD)

$6,8^{\star}$

Fonds africain de développement (FAD)

$6,2^{*}$

Banque asiatique de développement (ADB)

$1,1^{*}$

Fonds asiatique de développement (ADF)

$0,3^{*}$

Banque interaméricaine de développement (BID)

$1,5^{\star}$

Société d'investissement interaméricaine (JJC)

3. Autres organismes multilatéraux

Fonds international pour le développement agricole (FIDA)

Autres organismes

4. Organisations privées internationales

Remarque: Les chiffres indiqués dans le tableau représentent des versements, tandis que les astérisques signifient l'émission de reconnaissances de dettes irrévocables ( «notesu). Lindication simultanée d'un chiffre et d'un astérisque donne le montant des «notes vencaissées par le bénéficiaire et l'émission de nouvelles reconnaissances de dettes irrévocables.

Voici un aperçu de ces opérations:

\begin{tabular}{|c|c|c|}
\hline & 1984 & 1985 \\
\hline (1) & 54,9 & 70.6 \\
\hline Versements ( \&notes- encaissées) & 0,8 & 9,3 \\
\hline
\end{tabular}

Rappel: Le CAD estime les flux d’aide publique au développement en tenant compte des émissions, alors que la suisse se fonde sur les versements.

Source. DDA, Recueil de statistiques, octobre 1987. 
dont 50 millions environ sont payables, le reste étant constitué par du capital de garantie.

Les banques régionales de développement sont des instruments de la coopération multilatérale au développement. La Suisse considère que les activités de ces banques sont un complément indispensable des relations bilatérales. Les banques de développement représentent pour les pays les moins avancés une des sources les plus importantes de financement du développement. Leur rôle s'est même accru dernièrement car les flux de capitaux privés dans les pays en développement ont accusé un net déclin.

La Suisse fait partie de l'ADB depuis 1967, de la BID depuis 1976 et de la BAD depuis 1982; elle alimente aussi leurs fonds de développement. Les contributions versées à ces fonds sont prélevées sur le crédit-cadre pour la coopération et l'aide financière.

\section{Renforcement de la coopération}

Parmi les motifs qui plaident pour l'intensification de la coopération multilatérale au développement, le Conseil fédéral évoque dans son message la possibilité de soutenir par des programmes multilatéraux les pays en développement qui présentent les critères requis pour la coopération, mais qui ne sont pas des partenaires de la Suisse au niveau des programmes bilatéraux, car les capacités limitées de la Suisse en ce qui concerne le personnel et l'administration ne le permettent pas. De plus, l'aide multilatérale répond au principe de l'universalité. Le Conseil fédéral ajoute que l'aide multilatérale est mise à disposition des pays en développement sous une forme non liée, contrairement à bien des projets d'aide bilatérale. II faut cependant relever ici que les banques régionales de développement lient souvent l'octroi de crédits à certaines conditions, telles que des mesures d'ajustement économique, en particulier pour des crédits d'ajustement structurel.

D'autres avantages de l'aide multilatérale consistent à promouvoir la coopération entre pays en développement de même que la coordination de l'aide à un haut niveau international.

\section{Les tâches des banques de développement}

Les banques régionales de développement ont été créées dans les années soixante sur le modèle de la Banque mondiale.*

Elles représentent une source importante pour l'octroi de crédits à long terme, à des fins de développement. Elles soutiennent notamment des projets qui ont une utilité économique ou sociale importante (projets d'infrastructure), ainsi que des

- Début des activités: BID 1960; ADB et BAD, 1966. 
projets qui visent directement les secteurs de la population les moins favorisés. L'objectif de leur politique consiste à mettre sur pied des institutions locales viables et à promouvoir ainsi un développement autonome. Cette politique va dans le sens de la Loi suisse de 1976 sur la coopération internationale au développement et l'aide humanitaire de 1976.

Les banques régionales de développement revêtent une importance particulière au vu de l'évolution économique actuelle: entre 1980 et 1985, le flux de ressources privées destinées aux pays en développement a diminué de $75 \%$ en termes réels. (Message, p. 11**). Le Conseil fédéral estime que, à court terme, une augmentation n'était guère probable. Par conséquent, l'augmentation des crédits des banques régionales de développement représente une contribution importante à la solution des crises actuelles de balance des paiements.

Les seuls critères utilisables pour l'octroi de crédits doivent être objectifs et de nature économique et financière, conformément aux statuts. La Suisse s'est prononcée en 1986 en faveur du déblocage d'un crédit agricole de la BID au Nicaragua, crédit que les Etats-Unis avaient bloqué pour des raisons politiques; elle avait agi de même dans le cas d'un crédit bloqué pour le Chili.

Les prêts des banques régionales de développement sont exclus des négociations sur le rééchelonnement de dettes. Les banques ont une position relativement solide sur les marchés de capitaux, qui tient à une politique commerciale prudente et au capital de garantie des pays membres en monnaies convertibles (principalement des pays industrialisés).

Le marché suisse des capitaux représente une source de financement importante pour les banques régionales de développement. Jusqu'à fin 1985, le total des emprunts nets en capital de la BID en Suisse a représenté 1,686 millions de dollars, celui de l'ADB 1,016 millions de dollars. La BAD a contracté pour la première fois en 1984, puis en 1986, des emprunts d'une valeur de 100 millions de francs (Message, p. 55*").

\section{Les Fonds de développement}

Les trois banques régionales de développement gèrent aussi un fonds de développement de montants non remboursables fournis par les membres économiquement plus forts. Les banques utilisent ces fonds pour accorder des prêts à des conditions préférentielles aux pays les plus pauvres de leur région. La contribution suisse à ces fonds n'est pas prélevée sur le crédit-cadre dont il est question ici (réservé uniquement aux augmentations de capital), mais sur le crédit de programme pour la coopération technique et l'aide financière.

* Les indications de pages correspondent à la version allemande. 


\section{L'attitude de la Suisse}

La Suisse est représentée dans les banques régionales de développement, de temps à autre au Conseil des gouverneurs et, par rotation, au Conseil exécutif.

Lors des négociations sur la septième augmentation du capital de la BID, la Suisse s'est engagée à faire bénéficier de nouveau les groupes à faible revenu de $50 \%$ des prêts concernant des projets.

Dans le cadre de la BAD, la Suisse s'est prononcée en faveur d'une limite d'endettement pour garantir la sécurité des emprunts accordés; sa position a été suivie par ses partenaires. Lors de la session annuelle de la BAD, le gouverneur suisse, $M$. E.Roethlisberger, a réclamé la création d'un système d'emprunts et de remboursements en devises, de façon à protéger la banque des pertes sur les taux de change. Il a en outre critiqué le fait que la banque soutient parfois des projets mal préparés et peu rentables.

Grâce à l'appartenance de la Suisse à ces institutions, les entreprises suisses peuvent répondre aux appels d'offres internationaux pour des projets cofinancés par les banques de développement. Entre 1983 et 1985, le montant qui est revenu en Suisse sous forme de commandes relatives à des projets de la BID était trois fois et demi supérieur à celui que la Suisse avait versé à la BID. (Message, p.54**).

\section{Augmentation de capital}

Au moment de la rédaction du message, les négociations sur la septième augmentation de capital de la BID n'avaient pas encore abouti. La dernière augmentation de capital de l'ADB datait de 1983 (pour la période 1983-1987). Selon les calculs de la banque, une quatrième augmentation de capital ne sera pas nécessaire avant 1989. II faut ajouter ici que les trois banques de développement se trouvent dans une phase de remboursement des crédits accordés avant 1980, ce qui renforce leur situation financière. Ces remboursements seront une source importante de nouveaux crédits au cours des prochaines années.

En ce qui concerne la BAD, les négociations sur la quatrième augmentation de capital sont en cours. Une augmentation substantielle s'avère nécesssaire; en effet, comparés aux programmes d'emprunts de la BID et de l'ADB, ceux de la BAD ont été jusqu'à présent fort modestes. L'Afrique a un grand besoin de capitaux qui sera en partie couvert par une importante augmentation du capital. Lors de l'Assemblée générale de juin 1987, il a été décidé de procéder à une augmentation de capital de $200 \%$.

Le Conseil fédéral prévoit pour la Suisse les incidences financières suivantes:

** Les indications de pages correspondent à la version allemande. 
Participation au capital à verser

\begin{tabular}{rcrcrc} 
& \multicolumn{1}{c}{ BID } & \multicolumn{2}{c}{ ADB } & \multicolumn{2}{c}{ BAD } \\
$\%$ & Mio Fr. & $\%$ & Mio Fr. & $\%$ & Mio Fr. \\
4,5 & 5,4 & 5,0 & 12,0 & 7,0 & 20,0 \\
95,5 & 112,5 & 95,0 & 230,0 & 33,0 & 258,0 \\
100,0 & 117,9 & 100,0 & 242,0 & 100,0 & 278,0
\end{tabular}

En mars 1987, le Conseil fédéral a également décidé, dans le cadre de la quatrième augmentation du Fonds de développement de la Banque asiatique, de mettre à disposition un montant non remboursable de 87,6 millions de francs.

Le Conseil des Etats a examiné le projet sur les banques régionales de développement lors de sa session de mars 1987. Le crédit-cadre a été approuvé par 27 voix contre 4.

\section{Les critiques}

La Déclaration de Berne considère que la participation de la Suisse à l'augmentation de capital des banques régionales de développement pose problème. Certes, ces banques financent davantage de petits projets et de projets adaptés aux besoins des plus pauvres; toutefois, elles continuent à financer dans une large mesure des projets mammouth dans les secteurs de l'énergie, des communications et des transports (selon la DB). La contribution renouvelée de la Suisse à l'augmentation du capital, prélevée sur le budget de l'aide au développement, diminue de plusieurs millions les possibilités de coopération directe et bilatérale.

La DB, de même que R.Gerster"**, critiquent le manque d'objectivité du message, en ce sens qu'il met au premier plan les aspects positifs des banques de développement, passant systématiquement sous silence les grands projets qui sont plus "douteux". Le rôle des banques de développement, par exemple dans le cas du grand barrage El Cajon au Honduras, n'est pas évoqué.

\section{Sources}

Message sur la participation de la Suisse à l'augmentation de capital des banques de développement interaméricaine, asiatique et africaine, ainsi que sur l'adhésion de la Suisse à l'Agence multilatérale de garantie des investissements, 26 novembre 1986.

DFEP, Communiqué de presse du 16 mars 1987

NZZ, 12.6.1987

** DB, "Modemisierungshilfe oder Entwicklungszusammenarbeit?", Article pour la presse, 17.1.1987.

R.Gerster, Coopération suisse au développement :"Weder Routine noch Kurswechsel", Documentation i3m, juin 1987. 


\section{Mesures économiques et commerciales}

Les mesures de politique économique et commerciale de la coopération technique se répartissent en cinq domaines: aide à la balance des paiements, crédits mixtes, mesures relatives aux matières premières, promotion du commerce en faveur des pays en développement et incitation à l'investissement de ressources économiques privées pour l'industrialisation de ces pays. En ce qui concerne l'engagement de fonds pour les mesures précitées, la responsabilité incombe à I'OFAEE (Office fédéral des affaires économiques extérieures), dépendant du Département fédéral de l'économie publique.

En 1986, les dépenses pour les mesures de politique économique et commerciale se sont montées à 71,8 millions de francs (en 1985: 73,2). Les attributions de fonds selon les types de mesures se présentent comme suit :

1986

Aide à la balance des paiements

Crédits mixtes

Promotion commerciale

Industrialisation

Produits de base

Total mio de francs

43,5

23,4

2,3

2,0

0,6

71,8
$\%$

60

33

3

3

1

100

Source: DDAOFAEE, Rapport annuel 1986

Sur l'ensemble des fonds de l'OFAEE répartis bilatéralement, $67,2 \%$ sont allés à l'Afrique, et plus particulièrement au Sénégal et à la Guinée. En Asie, 10\% des fonds ont été attribués à la Thaïlande, à l'Inde et au Sri Lanka; $17,1 \%$ des fonds ont été destinés à l'Amérique latine, plus précisément à la Bolivie et au Honduras.

Dans ce secteur, les obligations sont financées par le troisième crédit-cadre que le Parlement a approuvé le 8 octobre 1986. Celui-ci a une durée de trois ans et a été engagé à partir de la deuxième moitié de 1987; il représente 430 millions de francs. Le crédit-cadre précédent était de 350 millions de francs et a été porté ultérieurement à 450 millions dans le cadre des dispositions de création d'emplois.

Les dépenses effectuées dans le cadre des cinq secteurs d'activité se répartissent comme suit :

Crédits mixtes

Aide à la balance des paiements

Produits de base

Promotion commerciale

Industrialisation
240 millions de francs

120 millions de francs

50 millions de francs

10 millions de francs

10 millions de francs 
Le rapport annuel 1986 produit conjointement par la DDA et l'OFAEE au sujet de la coopération technique donne des explications assez détaillées sur l'aide à la balance des palements. Les premiers projets d'aide à la balance des paiements (Soudan I, 1981; Madagascar I, 1982; Ghana I, 1984) avaient le caractère de mesures d'urgence destinées à financer des programmes d'ajustement macroéconomiques spécifiques; dans une deuxième phase, ce type d'aide a été accordée pour financer des mesures d'ajustement structurel (Madagascar II et III, 1984-85; Ghana II et III, 1985-86). Les conditions actuelles d'octroi de l'aide suisse en matière de balance des paiements sont les suivantes :

- difficultés au niveau de la balance des paiements,

- programme d'ajustement structurel détaillé.

- soutien international exprimé face à ce programme.

L'aide à la balance des paiements a pris davantage d'importance au cours des dernières années en raison du nombre croissant d'interventions d'urgence et de programmes d'ajustement structurel. Cette évolution se traduit clairement dans les chiffres, puisque ce type d'aide représente 120 millions de francs $(28 \%)$ dans le troisième crédit-cadre, mais qu'il était seulement de 70 milions de francs $(20 \%)$ dans le deuxième.

En 1986, quatre projets d'aide à la balance des paiements sont entrés en vigueur; pour la première fois, un pays latino-américain, la Bolivie, a obtenu ce genre d'aide de la Suisse (10 millions de francs). Par ailleurs, le projet Ghana III représentait 10 millions de francs; ces deux projets étaient bilatéraux. Les projets de cofinancement avec l'AID ont concerné la Guinée (10 millions de francs) et le Sénégal (13,5 millions de francs).

A la fin de l'année 1986, la Suisse avait accordé une aide à la balance des paiements dans quinze cas en tout, pour un montant total de 172,7 millions de francs. Au cours du premier semestre de 1987, aucun accord portant sur l'aide à la balance des paiements n'a été signé.

Dans le domaine des crédits mixtes, la Suisse a conclu quatre accords en 1986:

- accord sur un deuxième crédit mixte à la Tunisie (60 millions de francs); la part de la Confédération se monte à 21 millions de francs.

- l'Indonésie a obtenu un crédit mixte de 153 milions de francs pour l'agrandissement de sa centrale thermique de Belawan. (Part de la Confédération : 51 millions de francs).

- crédit mixte de 60 millions de francs à la Jordanie, dont une participation de la Confédération de 20 millions de francs.

- crédit mixte de 22,2 millions de francs à la Colombie (participation de la Confédération: 7,4 millions de francs).

Depuis 1977, la Suisse a conclu 21 accords de crédits mixtes pour une valeur totale de 1075 millions de francs, dont une participation de la Confédération d'environ 402 millions de francs. 
Dans le domaine des matières premières sont englobées diverses activités visant à soutenir les efforts des pays en voie de développement pour commercialiser leurs matières premières. Dans la période examinée ici, un montant de 950'000 francs pour deux ans a notamment été versé à l'Institut international du coton à Bruxelles, afin de promouvoir l'utilisation du coton. De même, l'organisation internationale du jute, à Dhaka, a reçu un soutien financier de 850'000 francs; d'autres institutions dans le domaine des matières premières ont également obtenu une aide financière.

Dans le secteur de la promotion du commerce, les organisations et activités suivantes ont bénéficié d'un soutien (la liste n'est pas exhaustive) :

- Centrale suisse pour la promotion commerciale;

- Projet de la CNUCED visant à aider les pays en voie de développement lors de l'introduction du traitement des données dans le secteur des douanes;

- Financement d'un séminaire de la CNUCED sur les préférences tarifaires à l'intention des pays africains les moins avancés.

\section{Alde humanitaire}

La DDA est responsable des mesures prises en matière d'aide humanitaire, y compris d'aide alimentaire. En 1986, l'aide alimentaire s'est montée à 148,5 millions de francs, contre 149,4 l'année précédente. Ce programme d'aide comporte trois secteurs d'activité :

Contributions aux organisations internationales et aux oeuvres d'entraide nationales 1985 1986

Aide alimentaire

Corps suisse d'aide en cas de catastrophes (ASC) Total

$\begin{array}{rr}83,7 & 63,1 \\ 53,3 & 71,9 \\ 11,5 & 14,4 \\ 148,5 & 149,4\end{array}$

Environ $30 \%$ des dépenses au titre de l'aide humanitaire ont concerné l'Afrique. Ce programme d'aide est financé par un crédit-cadre spécial de $\mathbf{4 4 0}$ millions de francs (de juillet 1985 à juillet 1988).

Au cours de la période du rapport, la Suisse a consacré à nouveau une part importante de son budget, à savoir 40 millions de francs (45 millions en 1985) à l'alde aux réfugiés. En raison de la guerre en Afghanistan, de nombreux réfugiés se trouvent au Pakistan et en Iran, où ils reçoivent soins et assistance. Cependant, l'aide aux réfugiés a surtout été dispensée à l'Afrique où la situation des réfugiés s'est dégradée suite à divers conflits internes (Somalie, Soudan). La DDA a accordé à cet effet la somme de 23,4 millions de francs en 1986 au Haut-Commissariat des Nations Unies pour les réfugiés. 
Dans le domaine de l'aide alimentaire, les dépenses ont été en 1986 inférieures de $\mathbf{2 0}$ milions au montant de l'année d'avant, alors que la famine en Afrique avait nécessité la mobilisation de ressources considérables. En 1986, la Suisse a livré ou financé 54'086 tonnes de denrées, notamment des céréales et des produits laitiers suisses. Les céréales ont fait l'objet à $90 \%$ d'opérations triangulaires, c'est-à-dire qu'elles ont été achetées dans des régions de production excédentaire du Tiers Monde et revendues aux régions souffrant de pénurie. Actuellement, divers aspects de l'aide alimentaire sont examinés, en particulier le contrôle de l'aide; ces questions devront être traitées en détail dans le message relatif à un nouveau crédit-cadre.

Les contributions aux organisations internationales: Le Comité international de la Croix-Rouge (CICR) fournit une part importante de l'aide humanitaire; la DDA a contribué en 1986 à cette action par un montant de 53,2 millions de francs. Les autres organisations qui contribuent à ce programme sont la Croix-Rouge suisse, le Haut-Commissariat des Nations Unies pour les réfugiés, l'Organisation des Nations Unies pour l'aide en cas de catastrophes (UNDRO), le Programme alimentaire mondial (PAM), l'oeuvre d'entraide des Nations Unies pour les réfugiés palestiniens.

Les oeuvres suisses d'entraide ont reçu 27 milions de francs à titre de contribution pour leurs programmes d'aide humanitaire.

Le Corps suisse d'alde en cas de catastrophes (ASC) apporte une aide d'urgence en cas de catastrophes naturelles et de conflits. L'aide est fournie très rapidement, sous forme d'actions directes menées par une équipe de bénévoles qualifiés. En 1986, les interventions directes de l'ASC se sont chiffrées à 7,6 millions de francs (7,7 en 1985), et ont eu lieu dans 18 pays en développement ainsi qu'en Grèce (tremblement de terre). Récemment, la procédure disciplinaire intentée contre le chef de l'ASC, M.Edouard Blaser, a suscité de vives réactions. La Commission d'enquête du Conseil des Etats avait constaté en été 1986 des divergences entre la DDA et l'ASC qui touchaient principalement aux limites des compétences. Après que M.Blaser eut annoncé en décembre 1986 sa démission pour mi-87, il fut relevé de ses fonctions à la fin du mois. Le bruit a couru que le licenciement de M.Blaser faisait suite à l'annonce d'une procédure disciplinaire. Pendant six mois, le "cas Blaser" a été l'objet de débats publics et parlementaires.

Les reproches formulés à M.Blaser portaient sur son manque de coopération avec la DDA, la non observation des directives et de nombreux excès au niveau du pouvoir décisionnel. Une enquête disciplinaire menée par un organe indépendant a été suspendue en août 1987, après que l'on eut constaté certains manquements aux devoirs.

L'affaire a montré clairement que l'aide d'urgence et la coopération au développement qui en découle, à moyen et à long terme, ne sont pas assez bien définies. Le Conseil fédéral a décidé le 29 avril 1987 de confier au futur chef de I'ASC, qui portera le titre de "Délégué pour l'aide en cas de catastrophes à l'étranger", la direction du département de l'aide humanitaire. Les "sections 
indépendantes" de l'ASC et la section de l'aide humanitaire et de l'aide alimentaire seront à nouveau, comme c'était le cas avant août 1986, réunies en un seul département au sein de la DDA. Le caractère de milice du corps de volontaires doit être conservé et il s'agit de garantir, par le biais d'une ordonnance, que le Délégué pour l'aide en cas de catastrophes à l'étranger continue à disposer d'une compétence financière d'un million de francs en cas d'actions urgentes.

\section{Les débats parlementaires}

Au cours de la période sous revue, un des points essentiels des débats au sein du Parlement et des différentes commissions a été l'examen du rapport sur la dernière décennie d'aide publique au développement et des messages sur le nouveau crédit-cadre pour la coopération technique et l'aide financière ainsi que sur les augmentations de capital des banques régionales de développement. (Ces différents débats sont résumés dans les chapitres correspondants).

Commission consultative de la coopération internationale au développement: Cette commission créée en 1976 a pour tâche de conseiller le Conseil fédéral et l'Administration fédérale en matière de coopération au développement et d'aide humanitaire. Elle se compose de 19 représentants des principaux partis politiques, des oeuvres d'entraide, de l'industrie, des banques et des syndicats. Les écoles supérieures et les média sont également représentés au sein de cette commission qui siège quatre fois par an.

Le point essentiel de la session de novembre 1986 a consisté à examiner les aspects écologiques de la coopération technique, en particulier les possibilités de mettre un terme à la destruction des ressources naturelles. L'on a aussi examiné la proposition consistant à étudier les projets et opérations de la DDA et de l'OFAEE sous l'angle de leur compatibilité avec l'environnement. Une étude de l'Institut de géographie de l'Université de Berne a servi de base à la discussion. La Commission a recommandé aux offices fédéraux responsables de la coopération technique de renforcer les mesures en vue d'intégrer systématiquement les contingences de l'environnement dans les projets de développement.

Lors de la séance de février 1987, un débat a eu lieu, avant les délibérations sur le message relatif au nouveau crédit-cadre pour la coopération technique et l'aide financière, au sujet de la décision de relever M.Blaser de ses fonctions de chef de I'ASC. La commission consultative qui est aussi compétente en matière d'aide humanitaire est chargée de coordonner l'aide d'urgence de l'ASC et l'aide au développement de la DDA. Elle a suggéré d'intégrer l'ASC à la structure de la DDA.

En mai, une séance a réuni la commission consultative pour la coopération technique et la commission de politique économique extérieure au sujet de la conférence de la CNUCED. 
De même, la commission consultative pour la coopération technique a discuté en mai du rapport sur la coopération au développement de 1976 à 1985.

La Commission du Conseil national pour les affaires étrangères s'est également penchée sur le cas Blaser. Elle a aussi entendu des experts sur la question des sanctions à l'encontre de l'Afrique du Sud et de l'attitude de la Suisse à ce sujet. Le conseiller national M. Rechsteiner (PS) a présenté à la commission son initiative parlementaire, consistant à demander qu'un arrêté fédéral soumette au "courant normal" toutes les exportations de capitaux vers l'Afrique du Sud et les transactions concernant l'or sud-africain, ce qui signifierait le gel des transactions au niveau actuel. La Commission propose au Parlement le rejet de l'initiative Rechsteiner.

La Commission des pétitions a discuté de la pétition "Pour un commerce équitable avec le Tiers Monde" au cours de deux séances (novembre 1986 et mars 1987). La pétition des oeuvres d'entraide a été remise aux autorités en octobre 1986 (Cf. article dans l'Annuaire 1986). La Commission est parvenue à la conclusion que la pétition soulevait des questions de fond et regrette que la Confédération ne dispose pas d'un concept précis en ce qui concerne les relations commerciales de la Suisse avec les pays en développement. Elle adresse au Conseil fédéral un postulat réclamant un rapport sur les relations commerciales que la Confédération entretient avec le Tiers Monde et sur ses efforts pour promouvoir les produits des pays en développement respectant certains critères sociaux et écologiques.

En outre, le Conseil fédéral a répondu à l'interpellation Belser (PS) portant sur l'aide multilatérale au développement. L'interpellateur a demandé au Conseil fédéral quelles étaient les perspectives de ce type d'aide pour les cinq années à venir, compte tenu de la tendance à privilégier l'aide multilatérale par rapport à l'aide bilatérale. II s'est également enquis du maintien de la répartition des programmes d'aide : deux tiers d'aide bilatérale et un tiers d'aide multilatérale. Le Conseil fédéral expose à nouveau dans sa réponse les motifs de maintenir la répartition actuelle. L'évaluation des projets d'aide multilatérale aurait donné des résultats satisfaisants, de même que pour les projets bilatéraux.

Au cours de la session de juin 1987, deux propositions ont été lancées : un postulat (Longet) enjoint au Conseil fédéral de présenter un rapport sur les flux financiers entre la Suisse et le Tiers Monde et sur les relations d'échange ainsi que sur les possibilités pour la Suisse d'agir sur ces deux facteurs.

Une motion (Gurtner) demande au Conseil fédéral d'intégrer, au sein de la DDA, un poste pour respecter les besoins des femmes dans la coopération au développement, notamment en garantissant leur engagement direct dans les négociations, en tenant compte de leurs besoins, et en élaborant des projets de recherche spécifiques concernant les femmes du Tiers Monde de façon à recueillir les informations nécessaires et améliorer leur situation. (Cf. aussi le dossier "Femmes et développement" dans la deuxième partie de l'Annuaire). 
Les Commissions financières des deux Consells traitent régulièrement des questions de coopération au développement. Au cours de la période envisagée ici, la délégation financière composée de membres des deux commissions a discuté notamment des avantages et des inconvénients de la coopération avec des organisations non gouvernementales (ONG). La DDA envisage d'intensifier son engagement dans ce domaine au cours des années à venir, car les ONG fournissent la plupart du temps une aide plus rapide, plus efficace et souvent plus durable.

\section{Inde}

Au cours de la période sous revue, la Commission financière fédérale a poursuivi, de concert avec la délégation financière, l'examen des projets à l'étranger; elle a notamment examiné certains projets de l'aide suisse au développement en Inde. En premier lieu, il s'agissait d'évaluer un gros projet d'aide financière émanant du domaine du crédit agricole (fonds engagés par la Confédération: environ 140 millions de francs). De l'avis de la Commission, ce projet comporte des aspects criticables. En effet, les dispositions contenues dans les contrats entre l'Inde et la Suisse relativement au prêt productif d'intérêts et remboursable en monnaie indienne ainsi qu'à l'utilisation ultérieure de ces fonds par le gouvernement central de New Delhi, laissent encore bien des questions importantes sans réponse. D'une manière générale, le travail réalisé avec l'Inde est considéré comme positif. La Commission financière suggère qu'il serait peut-être plus avantageux de mettre en oeuvre des programmes d'aide bilatérale en Inde, compte tenu des expériences faites dans ce pays, programmes qui apporteraient une aide à ceux qui en ont réellement besoin.

\section{Coopération au développement des organismes privés}

Les oeuvres d'entraide privées, les missions et les autres organisations privées représentent une part importante de la coopération suisse au développement, particulièrement au niveau de l'aide bilatérale. Les dépenses sont couvertes d'une part par des dons et des cotisations de membres et d'autre part par des subventions de la Confédération.

En 1986, les versements des oeuvres d'entraide (sans les subventions de la Confédération mais en comptant les contributions des cantons et des communes) pour des projets de coopération technique et d'aide humanitaire se sont montés à 131,2 millions de francs (146,1 en 1985). Le tableau ci-après présente les contributions des différentes organisations qui ont consacré en 1986 plus d'un million de francs de dons privés en faveur de projets de coopération technique et 


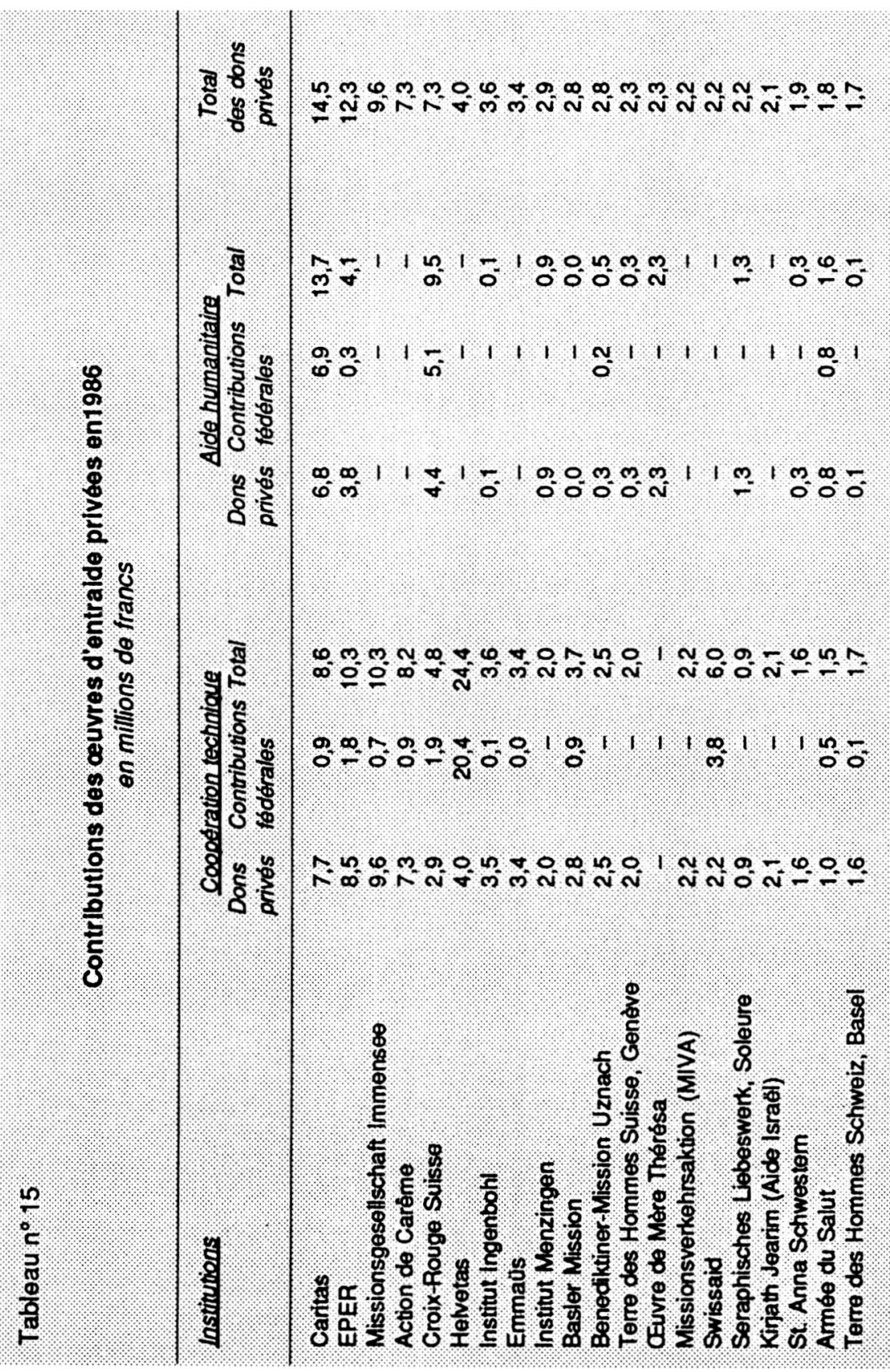




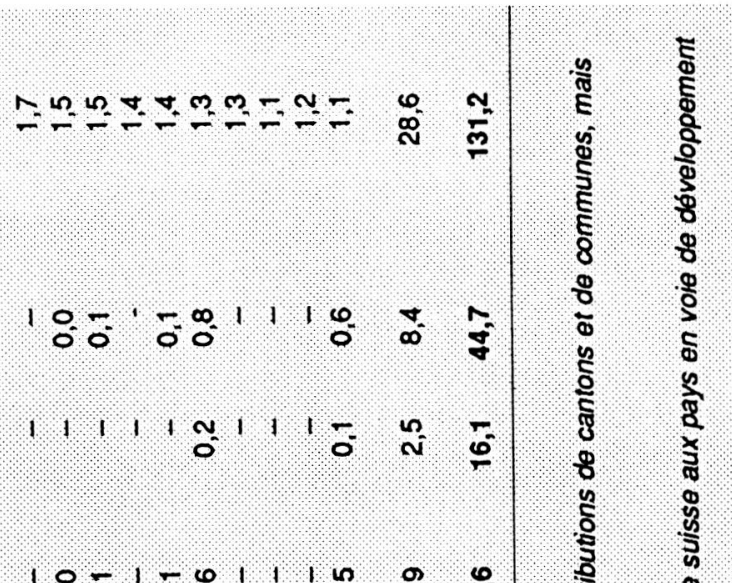

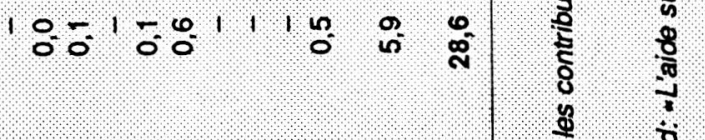

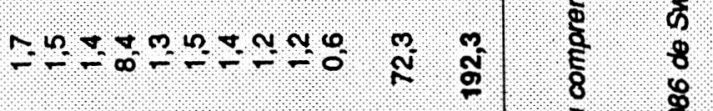

$$
\begin{aligned}
& \text { ól. }
\end{aligned}
$$

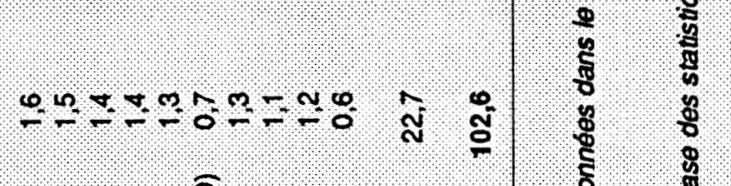

$$
\begin{aligned}
& \text { ชิ } \\
& \text { 5 } 8
\end{aligned}
$$

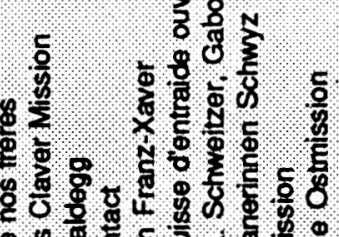

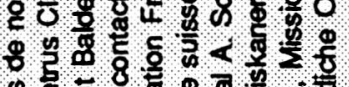

$$
\begin{aligned}
& \text { of }
\end{aligned}
$$

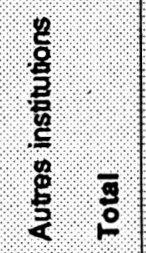

$$
\begin{aligned}
& \text {. } 8 \\
& \text { की है : ? } \\
& \frac{5}{5} \frac{8}{8}, 5 \\
& \text { है है है क्ष } \\
& \text { (3) } 8 \text {. } 8 \text {. }
\end{aligned}
$$

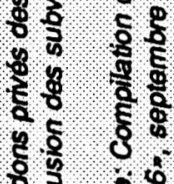

$$
\begin{aligned}
& \text { पै द. } \\
& \text { - } \stackrel{0}{6} \%
\end{aligned}
$$


d'aide humanitaire. En 1986, les dons privés destinés à la coopération technique ont totalisé 102,6 millions de francs et ceux destinés à l'aide humanitaire, 28,6 millions de francs.

De plus, la DDA a versé une contribution de 96,7 millions de francs aux oeuvres d'entraide (en 1985: 92,2); selon le rapport annuel de la DDA, celles-ci ont utilisé ce montant pour leurs actions directes (41,5 millions de francs) et pour des projets en régie (55,2 millions de francs). Ce sont Helvetas et Intercooperation qui ont reçu les contributions fédérales les plus importantes (26,1 millions de francs et 24,1 millions de francs respectivement).

Outre les activités reliées aux projets proprement dits, les organisations privées oeuvrant dans le domaine de la coopération technique mènent aussi un travail d'information auprès du public suisse. Par rapport à ce que diffusent les grandes agences de presse, les organisations privées fournissent des informations différentes et plus détaillées sur les pays en voie de développement. La Déclaration de Berne est particulièrement engagée dans ce travail d'information; elle a organisé une rencontre en novembre 1986 sur les exportations d'entreprises pharmaceutiques suisses dans le Tiers Monde, sous le titre "Médicaments chez nous et Tiers Monde - surplus et pénurie". En mai 1987, un colloque "Etats-Unis/ Suisse/tiers Monde" s'est tenu à Fribourg à l'occasion duquel l'on a abordé la question "De quelle Suisse le Tiers Monde a-t-il besoin?".

Chaque année ont lieu à l'IUED, à Genève, les "Rencontres des média NordSud". En avril 1987 il y a eu le concours d'émissions de télévision du monde entier sur la politique de développement et un colloque sur le thème "Les nouvelles technologies de communication et les relations Nord-Sud".

De nombreuses oeuvres d'entraide peuvent faire un véritable bilan de leurs expériences, certaines oeuvrant depuis 20 ou 25 ans dans le domaine de la coopération au développement. La centrale d'importation de marchandises du Tiers Monde, OS3, a fêté ses 10 ans d'existence. Cette centrale cherche à établir des relations commerciales plus équitables avec le Tiers Monde et informe le public suisse sur ses partenaires commerciaux dans les pays en voie de développement, leurs conditions de vie et de production, la situation du marché des matières premières. OS3 livre aux Magasins du Monde dans toute la Suisse des produits provenant des pays en développement.

Les oeuvres d'entraide ne dissocient pas leur activité des conditions politiques; l'EPER (Entraide protestante suisse) l'a prouvé à fin 1986 en renonçant à collaborer avec l'Union de banques suisses vu que celle-ci est très engagée en Afrique du Sud.

\section{Remarques}

1. Comité du développement de l'OCDE. D'après les estimations de l'OCDE, la part de la coopération suisse au développement représente $0,30 \%$ du PNB. Cette différence est due à une computation différente des "Notes" (reconnaissances de dettes) que la Suisse n'enregistre qu'au moment du remboursement effectif alors que l'OCDE les comptabilise dès leur émission. 


\section{Sources}

DDAOFAEE, Rapport annuel sur la coopération suisse au développement, 1986.

DDA, Service des statistiques.

Rapport sur la politique économique extérieure 1986.

Conseil national, 87.502, 87.440, 86.268.

Conseil des Etats, 86.578.

Rapport de la Délégation financière des conseillers fédéraux sur leur activité en 1986/87, présenté aux commissions financières du Conseil national et du Conseil des Etats, 27 avril 1987.

DFAE, Service de presse, 11.11.1986; 29.4.1987.

SBS, Le Mois, 9/86.

Document i3m, $1 / 87$.

NZZ, 26.8.1987.

Tages-Anzeiger, 24.11.1986, 15.5.1987. 